\title{
CHARACTERISATION OF PSEUDOMONAS AERUGINOSA RELATED TO BOVINE MASTITIS
}

\author{
Hye Rim PARK ${ }^{1 \dagger}$, Min Ki Hong ${ }^{2 \dagger}$, Sun Young HwANG ${ }^{1}$, Young Kyung PARK ${ }^{1}$, \\ Ka Hee Kwon ${ }^{1}$, Jang Won Yoon ${ }^{1}$, Sook SHIN ${ }^{1}$, Jae Hong KIM $^{3 *}$ and Yong Ho PARK ${ }^{1 *}$ \\ ${ }^{1}$ Department of Microbiology and Brain Korea 21 Program for Veterinary Science, \\ College of Veterinary Medicine, Seoul National University, Seoul, Korea; ${ }^{2}$ Viral Infec- \\ tious Disease Research Center, Korea Research Institute of Bioscience and Biotechnol- \\ ogy - University of Science and Technology, Daejeon, Korea; ${ }^{3}$ Laboratory of Avian Dis- \\ ease and Brain Korea 21 Program for Veterinary Science, College of Veterinary Medi- \\ cine, Seoul National University, San 56-1, Daehak-dong, Seoul 151-742, Korea
}

(Received 20 September 2012; accepted 2 April 2013)

Pseudomonas aeruginosa is one of the causative pathogens of bovine mastitis. Most $P$. aeruginosa strains possess the type III secretion system (TTSS), which may increase somatic cell counts (SCCs) in milk from mastitis-affected cows. Moreover, most of $P$. aeruginosa cells can form biofilms, thereby reducing antibiotic efficacy. In this study, the presence and effect of TTSS-related genotypes on increase of SCCs among 122 P. aeruginosa isolates obtained from raw milk samples from mastitisaffected cows and their antibiotic susceptibility at planktonic and biofilm status were investigated. Based on the presence of TTSS-related genes a total of $82.7 \%$ of the isolates were found to harbour $e x o U$ and/or $e x o S$ genes, including the invasive (exoU-/ exoS,$+ 69.4 \%$ ), cytotoxic (exoU+/exoS,$- 8.3 \%$ ) and cytotoxic/invasive strains (exoU+/ exoS+, 5.0\%). Milk containing exoS-positive isolates had higher SCCs than those containing exoS-negative isolates. The majority of isolates showed gentamicin, amikacin, meropenem and ciprofloxacin susceptibility at planktonic status. However, the susceptibility was decreased at the biofilm status. Based on minimum biofilm eradication concentration (MBEC)/minimum inhibitory concentration (MIC) ratios, the range of change in antibiotic susceptibility varied widely depending on the antibiotics (from $\geq 3.1$-fold to $\geq 475$.0-fold). In conclusion, most $P$. aeruginosa isolates studied here had a genotype related to increase in SCCs. The efficiency of antibiotic therapy against $P$. aeruginos $a$-related bovine mastitis could be improved by analysing both the MBEC and the MIC of isolates.

Key words: Pseudomonas aeruginosa, bovine mastitis, type three secretion system (TTSS), biofilm, minimum biofilm eradication concentration (MBEC)

\footnotetext{
'The first two authors contributed equally to this work. This paper was part of a dissertation submitted by Hye Rim Park to the Graduate School of Seoul National University in partial fulfillment of the requirements for the degree of Master in Veterinary Microbiology

"Corresponding authors; E-mail: kimhong@snu.ac.kr; Phone: 0082 (2) 885-6614; Fax: 0082 (2) 885-6614; E-mail: yhp@snu.ac.kr; Phone: 0082 (2) 880-1257; Fax: $0082(2) 871-7524$
} 
Pseudomonas aeruginosa, one of the pathogens frequently isolated from cows affected with mastitis, exists in various environments and can infect the cow through the teat canal. The microorganism carries several virulence factors such as exotoxin A, exoenzyme and protease, which bring about inflammatory responses and cell death. The resultant increase in milk somatic cell counts (SCCs) and decrease in other milk components such as fat, lactose, casein and calcium has caused economic losses in the dairy farming industry (Park et al., 2007). The bacterium also has intrinsic multidrug resistance that allows it to achieve further resistance. Moreover, most of the $P$. aeruginosa strains that have been isolated are strong biofilm producers that can reduce the efficacy of antibiotics and lead to chronic mastitis (Melchior et al., 2006).

The pathogen uses a wide range of virulence factors, including proteins capable of inducing toxicity, which effectively damage tissues (Lyczak et al., 2000). Some of these toxic effector proteins are injected into the cytosol of host cells through the type III secretion system (TTSS) and are involved in the disruption of epithelial surface and cell death. These TTSS proteins include cytotoxin (ExoU), ADP-ribosylating enzymes (ExoS and ExoT) and adenyl cyclase (ExoY). ExoU kills a variety of eukaryotic cells through its phospholipase activity. ExoT and ExoY are involved in adhesion, phagocytosis and systemic spread of the bacterial cells together with ExoS (Vance et al., 2005). ExoT and ExoY seem to play only minor roles in the pathogenesis, whereas ExoS and ExoU are known to determine specific patterns of pathogenesis (Engel and Balachandran, 2009). For example, invasive strains, which break the epithelial barrier and disseminate $P$. aeruginosa infection to other parts of tissues, usually possess the genes for ExoS, ExoT and ExoY, but lack the gene for ExoU. On the other hand, cytotoxic strains, which cause inflammation and tissue necrosis through their cytotoxic effect, typically possess the genes for ExoU, ExoT and ExoY, but lack the gene for ExoS (Fleiszig et al., 1997). These TTSS-expressing strains are capable of increasing the severity of disease (Roy-Burman et al., 2001) and have the ability to affect the SCCs and milk components by damaging mammary epithelial cells in the case of an intramammary infection. However, only a few studies have investigated the TTSS of $P$. aeruginosa isolated from bovine milk (Szmolka et al., 2012).

Biofilm-forming ability is another important characteristic of $P$. aeruginosa, which influences the pathophysiological pathway and antibiotic resistance of the bacterial cells, since the biofilm acts as a physiological barrier helping the bacteria to survive in a severe environment. In the mammary glands, biofilms facilitate the colonisation of the pathogens, their invasion into the epithelium and their evasion of the host's immune defence mechanism, since a biofilm provides a dense and protected environment that allows the bacterial cells to co-operate and interact in various ways (Oliveira et al., 2006). In addition, biofilms increase antibiotic resistance through multiple mechanisms like disturbing antibiotic 
penetration, general stress response and quorum-sensing. Under biofilm conditions, $P$. aeruginosa becomes up to 1,000 times more resistant to antibiotics than under planktonic conditions (Mah and O'Toole, 2001). However, most previous studies on $P$. aeruginosa isolated from cows affected with bovine mastitis focused on the activities of antibiotics against planktonic bacteria only.

In this study, TTSS-related genotypes of $P$. aeruginosa isolates obtained from the milk of cows affected with mastitis were screened and their influence on the SCCs and milk components (milk fat, milk protein and lactose) were studied. In addition, the antibiotic susceptibility profiles of $P$. aeruginosa of planktonic and biofilm status were also compared.

\section{Materials and methods}

\section{Raw milk sampling, analysis and bacterial isolation}

From January 2006 to December 2011, a total of 35,625 raw milk samples were collected from nine provinces in Korea. Raw milk samples were collected from cows that showed swollen udder quarters or entirely swollen udders, abnormal milk secretion and loss of appetite. In addition, cows whose milk showed flakes or clots were also used as a source of raw milk. The milk was collected aseptically from individual udder quarters of the cows, as described previously (Moon et al., 2007). The SCCs of each milk sample, which indirectly show the quantity of dead epithelial cells and neutrophils in the milk, and the percentage of milk components, including milk fat, milk protein and lactose, were analysed using a somatic cell counter (Bently Somacount 300, USA). Milk samples showing SCCs of over 500,000 cells $/ \mathrm{mL}$ were suspected to be mastitic milk (De Haas et al., 2005). In total, 3,996 samples were suspected as mastitic milk, and bacteria were isolated from these samples. Briefly, a loop of milk (approximately $10 \mu \mathrm{l}$ ) was inoculated onto a cetrimide agar plate (Difco, USA) and incubated at $42{ }^{\circ} \mathrm{C}$ for $24 \mathrm{~h}$. Putative $P$. aeruginosa isolates, acquired from the samples after using biochemical tests such as triple sugar iron (TSI) test, were confirmed by polymerase chain reaction (PCR) using the following $P$. aeruginosa-specific primers: PA-SS-F (5'-GGG GGA TCT TCG GAC CTC A-3') and PA-SS-R (5'-TCC TTA GAG TGC CCA CCC G-3') (Spilker et al., 2004). Overall, 462 P. aeruginosa isolates were confirmed and 122 isolates which were isolated alone without any other bacteria from raw milk samples were further examined.

\section{Detection of the TTSS-related toxin genes in $\mathrm{P}$. aeruginosa}

The presence of four TTSS genes (exoS, T, $Y$ and $U$ ) among the $122 P$. aeruginosa isolates was examined by PCR using gene-specific primers, as described previously (Ledbetter et al., 2009). The chromosomal DNA was ex- 
tracted with a DNeasy ${ }^{\circledR}$ blood and tissue kit (Qiagen Inc., USA) according to the manufacturer's instructions for Gram-negative bacteria. The $P$. aeruginosa strain ATCC 27853 was used as a reference strain for validating the PCR results.

Determination of minimum inhibitory concentration and minimum biofilm eradication concentration

Minimum inhibitory concentration (MIC) of planktonic status $P$. aeruginosa and minimum biofilm eradication concentration (MBEC) of biofilm status $P$. aeruginosa were compared using a Calgary Biofilm Device (Innovotech Inc., Canada) according to the manufacturer's instructions. MIC was determined as per the guidelines established by the Clinical Laboratory Standards Institute (CLSI, 2012) against the following 12 antibiotics: amikacin, aztreonam, cefoxitin, chloramphenicol, ciprofloxacin, colistin, erythromycin, gentamicin, kanamycin, meropenem, piperacillin and tetracycline. Escherichia coli ATCC 25922 and $P$. aeruginosa ATCC 27853 were used as quality control strains.

Isolates showing resistance to at least one agent in three or more antimicrobial categories were defined as multidrug-resistant (MDR) bacteria, and isolates showing resistance to at least one agent in all but two or fewer antimicrobial categories were classified into the extensive drug resistance (XDR) group. Isolates showing resistance to all agents in all antimicrobial categories were defined as pandrug-resistant (PDR) bacteria (Magiorakos et al., 2012).

Statistical analysis

Comparisons were made using one-way ANOVA and Student's $t$-test. Differences were judged significant at confidence levels of $>95 \%(\mathrm{P}<0.05)$. All data were analysed with SAS 9.3 for Windows (SAS Institute Inc, USA).

\section{Results}

Presence of the TTSS-related toxin genes among P. aeruginosa isolates from bovine mastitic milk

Of the 122 P. aeruginosa isolates, 99.2\% (121/122) possessed at least 1 of the 4 TTSS-related genes evaluated in this study (Table 1). One hundred $P$. aeruginosa isolates $(82.7 \%$ ) harboured exoU and/or exoS genes, including the non-cytotoxic/invasive strain (exoU-lexoS+, 69.4\%), cytotoxic/non-invasive strain (exoUt/exoS-, 8.3\%) and the cytotoxic/invasive strain (exoU+lexoS+, 5.0\%). The genes exoY and exoT were detected in 53 and 88 isolates (58.9 and $97.8 \%$ ) of invasive strains, respectively. 


\section{Table 1}

Distribution of the type III secretion system-related genotypes among Pseudomonas aeruginosa strains isolated from the milk of mastitis-affected dairy cows

\begin{tabular}{|c|c|c|c|c|c|c|}
\hline & exoU & $\operatorname{exoS}$ & exoY & exoT & No. of isolates & Percentage $(\%)$ \\
\hline \multirow{3}{*}{ Non-cytotoxic/Invasive strain (exoU-/exoS+) } & - & + & + & + & 46 & 37.7 \\
\hline & - & + & - & + & 36 & 29.5 \\
\hline & - & + & + & - & 2 & 1.6 \\
\hline \multirow{3}{*}{ Cytotoxic/Non-invasive strain (exoU+/exoS-) } & + & - & - & + & 8 & 6.6 \\
\hline & + & - & + & + & 1 & 0.8 \\
\hline & + & - & - & - & 1 & 0.8 \\
\hline \multirow{2}{*}{ Cytotoxic/Invasive strain $(\operatorname{exo} U+/ e x o S+)$} & + & + & + & + & 5 & 4.1 \\
\hline & + & + & - & + & 1 & 0.8 \\
\hline \multirow{3}{*}{ Non-cytotoxic/Non-invasive strain (exoU-/exoS-) } & - & - & - & + & 19 & 15.6 \\
\hline & - & - & + & + & 2 & 1.6 \\
\hline & - & - & - & - & 1 & 0.8 \\
\hline Total & & & & & 122 & 100 \\
\hline
\end{tabular}

\section{Table 2}

Milk component levels and somatic cell counts (SCCs) of milk samples from mastitis-affected cows

\begin{tabular}{|c|c|c|c|c|c|c|c|c|}
\hline & \multicolumn{2}{|c|}{ Milk fat (\%) } & \multicolumn{2}{|c|}{ Milk protein $(\%)$} & \multicolumn{2}{|c|}{ Lactose $(\%)$} & \multicolumn{2}{|c|}{$\operatorname{SCCs}\left(\times 10^{3}\right.$ cells $\left./ \mathrm{ml}\right)$} \\
\hline & Mean & SE & Mean & SE & Mean & SE & Mean & SE \\
\hline Cytotoxic/Invasive strain (exoUt/exoS +$)$ & 3.4 & 0.8 & 3.3 & 0.1 & 4.5 & 0.2 & 2449.0 & 236.0 \\
\hline Non-cytotoxic/Invasive strain (exoU-/exoS+) & 4.0 & 0.1 & 3.3 & 0.1 & 4.5 & 0.0 & 1509.6 & 148.4 \\
\hline Cytotoxic/Non-invasive strain (exoU+lexoS-) & 4.3 & 0.4 & 3.2 & 0.2 & 4.4 & 0.2 & 1294.2 & 431.4 \\
\hline Non-cytotoxic/Non-invasive strain (exoU-/exoS-) & 3.4 & 0.1 & 3.2 & 0.1 & 4.6 & 0.1 & 784.1 & 66.6 \\
\hline
\end{tabular}




\section{Relation between TTSS-related genotypes and bovine mastitic raw milk components}

The SCCs and milk components (milk fat, milk protein and lactose) in mastitic raw milk are summarised in Table 2, according to the 4 TTSS-related genotypes of $P$. aeruginosa isolated from samples of mastitic raw milk. No statistically significant differences were observed in milk components with respect to the TTSS-related genotypes of isolates $(\mathrm{P}=0.2,0.7$ and 0.6 for milk fat, milk protein and lactose, respectively). In terms of SCCs, significant differences were found among mastitic raw milk samples containing the cytotoxic/invasive strain, the non-cytotoxic/invasive strain and the non-cytotoxic/non-invasive strain $(\mathrm{P}<$ $0.05)$. However, there was no statistically significant difference between mastitic raw milk samples containing the cytotoxic/non-invasive strain and the samples containing the non-cytotoxic/non-invasive strain $(P=0.3)$. Samples containing the cytotoxic/invasive strain showed a significantly higher SCCs than those containing both cytotoxic/non-invasive and non-cytotoxic/invasive strains $(\mathrm{P}<0.05$; Fig. 1).

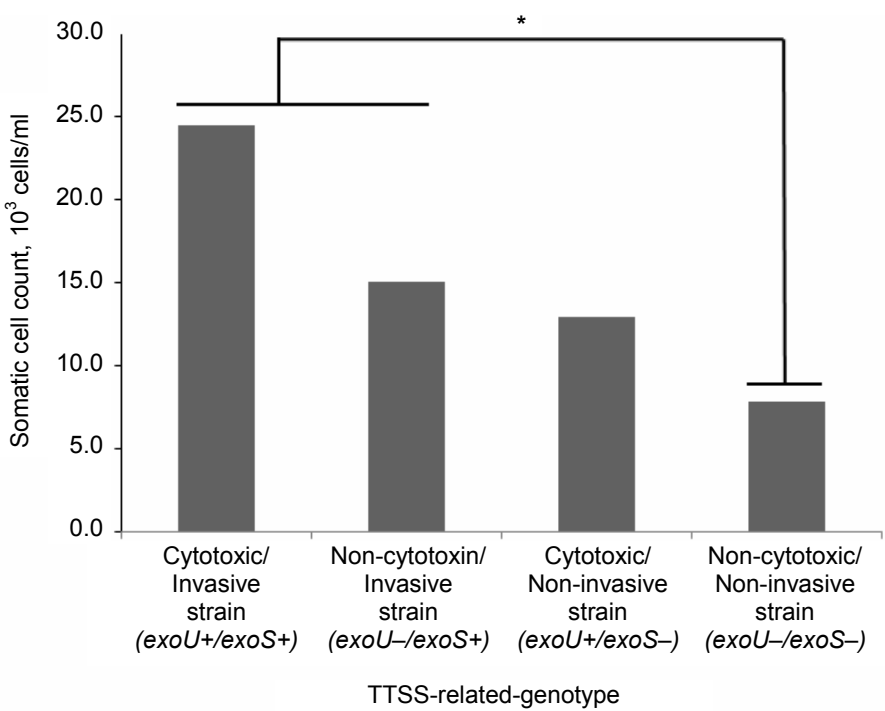

Fig. 1. Somatic cell counts of raw milk samples of mastitis-affected cows sorted according to the type III secretion system-related genotypes of Pseudomonas aeruginosa. Asterisk $\left(^{*}\right)$ denotes significant difference

Antibiotic susceptibility profile of planktonic and biofilm status $\mathrm{P}$. aeruginosa

The MIC of 122 isolates was measured and all isolates under planktonic cell status were found to be resistant to tetracycline and erythromycin (Fig. 2). Most of the planktonic status $P$. aeruginosa showed resistance or intermediate resistance to chloramphenicol $(99.2 \%$ of resistance and $0.8 \%$ of intermediate re- 
sistance), cefoxitin (93.4\% and 6.6\%), aztreonam (86.9\% and $11.5 \%)$, kanamycin $(65.6 \%$ and $34.4 \%)$, piperacillin $(63.9 \%$ and $0 \%)$, colistin $(94.3 \%$ and $5.7 \%)$ and gentamicin $(23.0 \%$ and $43.4 \%)$. Amikacin, meropenem and ciprofloxacin showed comparatively higher activity against planktonic status $P$. aeruginosa $(77.9 \%$, $86.9 \%$ and $98.4 \%$ of susceptibility, respectively) than other antibiotics. The MBEC of biofilm status $P$. aeruginosa was measured, and the MBEC/MIC ratio was calculated for each isolate (Table 3). The MBEC values of isolates against all antibiotics were higher than the MIC values. Even gentamicin, amikacin, meropenem and ciprofloxacin, which showed a relatively higher activity against planktonic status $P$. aeruginosa, had a MBEC value higher than $200 \mu \mathrm{g} / \mathrm{mL}$; therefore, their MBEC/MIC values were greater than $40(\geq 68.1, \geq 44.3, \geq 475.0$ and $\geq 407.1$, respectively).

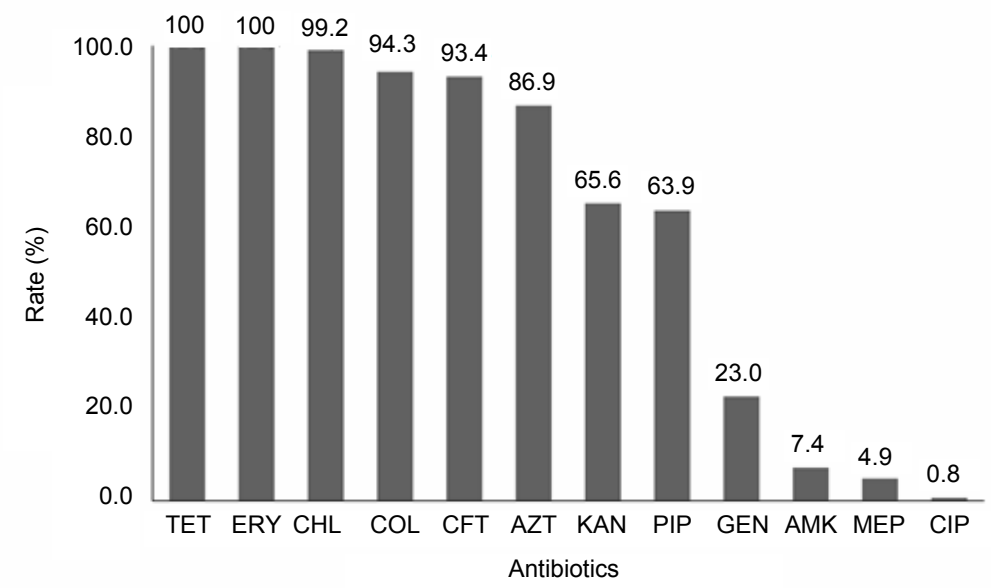

Fig. 2. Antibiotic resistance rates of the 122 bovine Pseudomonas aeruginosa isolates, based on the minimum inhibitory concentration (MIC) values measured by the broth dilution method.

TET, Tetracycline; ERY, Erythromycin; CHL, Chloramphenicol; COL, Colistin; CFT, Cefoxitin;

AZT, Aztreonam; KAN, Kanamycin; PIP, Piperacillin; GEN, Gentamicin; AMK, Amikacin; MEP, Meropenem; CIP, Ciprofloxacin

A total of $96.7 \%(118 / 122)$ and $16.4 \%(20 / 122)$ of planktonic status $P$. aeruginosa cells could be defined as MDR and XDR, respectively. No PDR isolate was detected in this study.

\section{Discussion}

In the dairy farming industry, reducing the SCCs is still a serious issue to be considered in the production of high-quality milk, although the incidence of mastitis in dairy cattle has decreased because of steady efforts to control mastitis. Moreover, high prevalence of subclinical and chronic mastitis due to recurrent 
infection has also been a persistent problem in the dairy farming industry (Hillerton and Kliem, 2002). The bacterium $P$. aeruginosa plays a critical role in the increase of SCCs and recurrent infection by causing cell death in the udder tissue and decreasing the efficiency of antibiotic therapy by forming biofilms (Melchior et al., 2006; Park et al., 2007).

Table 3

Minimum biofilm eradication concentration (MBEC)/minimum inhibitory concentration (MIC) ratios of 122 Pseudomonas aeruginosa isolates for 12 antibiotics

\begin{tabular}{|c|c|c|c|c|c|c|}
\hline \multirow{2}{*}{ Antibiotics } & \multicolumn{2}{|c|}{ MIC } & \multicolumn{2}{|c|}{ MBEC } & \multicolumn{2}{|c|}{ MBEC/MIC ratio* } \\
\hline & Mean & SE & Mean & SE & Mean & SE \\
\hline CIP & 0.5 & 0.0 & $\geq 206.7$ & 16.1 & $\geq 407.1$ & 31.8 \\
\hline MEP & $\geq 10.9$ & 5.9 & $\geq 410.2$ & 16.4 & $\geq 475.0$ & 38.6 \\
\hline GEN & $\geq 23.8$ & 7.2 & $\geq 442.0$ & 13.7 & $\geq 68.1$ & 4.3 \\
\hline AMK & $\geq 32.1$ & 8.1 & $\geq 460.5$ & 11.4 & $\geq 44.3$ & 3.0 \\
\hline COL & 111.4 & 15.4 & $\geq 471.3$ & 10.6 & $\geq 34.7$ & 3.0 \\
\hline KAN & 158.2 & 13.5 & $\geq 476.9$ & 10.0 & $\geq 11.9$ & 1.4 \\
\hline TET & $\geq 177.6$ & 16.8 & $\geq 490.0$ & 7.8 & $\geq 6.7$ & 0.5 \\
\hline ERY & $\geq 233.2$ & 14.1 & $\geq 497.3$ & 6.5 & $\geq 3.7$ & 0.4 \\
\hline CHL & $\geq 242.0$ & 15.6 & $\geq 512.0$ & 0.0 & $\geq 4.0$ & 0.4 \\
\hline PIP & $\geq 271.0$ & 20.0 & $\geq 507.8$ & 3.0 & $\geq 11.1$ & 2.0 \\
\hline AZT & $\geq 357.0$ & 20.1 & $\geq 508.9$ & 3.1 & $\geq 7.6$ & 1.1 \\
\hline CFT & $\geq 402.1$ & 16.6 & $\geq 509.9$ & 2.1 & $\geq 3.1$ & 0.5 \\
\hline
\end{tabular}

* MBEC/MIC ratio is a numerical value obtained by dividing MBEC by MIC for each isolate. As the maximum antibiotic concentration in this study was $512 \mu \mathrm{g} / \mathrm{mL}$, the same value was used in calculations with MIC or MBEC values higher than $512 \mu \mathrm{g} / \mathrm{mL}$, and these values have been presented with the inequality sign. CIP, Ciprofloxacin; MEP, Meropenem; GEN, Gentamicin; AMK, Amikacin; COL, Colistin; KAN, Kanamycin; TET, Tetracycline; ERY, Erythromycin; CHL, Chloramphenicol; PIP, Piperacillin; AZT, Aztreonam; CFT, Cefoxitin

A total of $99.2 \%$ of $P$. aeruginosa isolates in Korea have at least 1 of 4 TTSS-related genes, which is in harmony with earlier findings for human, environmental and bovine isolates of $P$. aeruginosa (Wiehlmann et al., 2007; Selezska et al., 2012; Szmolka et al., 2012). Of the 122 P. aeruginosa isolates, $68.8 \%$ of isolates were non-cytotoxic/invasive strain (exoU-lexoS +$)$, while only $8.2 \%$ of isolates were cytotoxic/non-invasive strain (exoUt/exoS-). Interestingly, 6 isolates possessing both exoU and exoS were detected, which is a notable finding since exoU and exoS appear to be mutually exclusive in different $P$. aeruginosa populations in human and environmental isolates (Wiehlmann et al., 2007; Selezska et al., 2012).

Since both cytotoxic and invasive strains are known to be able to cause inflammation and necrosis of infected epithelial cells, although severe inflammation and necrosis are associated with cytotoxic strains (Fleiszig et al., 1997), 
those were expected to cause an increase in SCCs in the case of an intramammary infection. Mastitic raw milk containing the cytotoxic/invasive strain, cytotoxic/non-invasive strain and non-cytotoxic/invasive strain did not show lower levels of milk components (milk fat, milk protein and lactose) than those containing the non-cytotoxic/non-invasive strain. In contrast, the SCCs was higher in mastitic raw milk containing the cytotoxic/invasive strain and the noncytotoxic/invasive strain than in the milk containing the non-cytotoxic/noninvasive stain. It is suggested that the TTSS might lead to an increase in SCCs by causing inflammatory reactions and recruiting inflammatory cells rather than by damaging the mammary epithelial cells. Cytotoxic/invasive strains possessing both exoS and exoU had a significantly higher effect on the increase in SCCs than the cytotoxic/non-invasive strains with exoU alone, and non-cytotoxic/ invasive strains with exoS showed a higher increase in SCCs than did the cytotoxic/non-invasive strains possessing exoU. It is also suggested that exoS might be involved in inflammatory reactions and necrosis to a greater extent than expected. Therefore, most of the $P$. aeruginosa isolates in this study, which had either exoS or exoU, should be considered as pathogenic for dairy cows.

Approximately $40 \%$ of mastitis cases develop into subclinical and chronic mastitis after failed therapy (Hillerton and Kliem, 2002). Most recurrent infections are often attributable to biofilm formation by bacteria. Since a biofilm matrix protects the bacterial cells from antibiotics and immune cells, bacteria in the biofilm can survive the action of antibiotics and result in recurrent infection.

In this study, more than $60 \%$ of planktonic status $P$. aeruginosa showed resistance to tetracycline, erythromycin, chloramphenicol, colistin, cefoxitin, aztreonam, kanamycin, piperacillin, gentamicin and amikacin which were approved for treatment of bovine mastitis and feed additives. In contrast, resistance rates for meropenem and ciprofloxacin, which were not permitted for veterinary use, were lower than 5\%. Since prescription from a veterinarian for antibiotics had not been obligatory until August 2013, more than 50\% of antibiotics consumed in farm animals have been used indiscriminately by non-professional persons. In addition, about $40 \%$ of antibiotics had been used as feed additives before such use was restricted (in July 2011). The amount of antibiotics used in farm animals was about 1,500 tons per year in Korea, which is 2 to 10 times higher than that of overseas countries considering the scale of livestock industry in Korea (Jung et al., 2008).

This causal relationship between antibiotic usage and antibiotic resistance was also reflected in the resistance rate for colistin, and the resistance rate confirmed in this study was higher than that found in a previous study (Sumathi et al., 2008). Like other antibiotics, colistin had been widely used in feed additives in Korea and the amount of polypeptides used in animals as feed additive had increased steadily (from 34 tons in 2001 to 46 tons in 2007) until the restriction. High colistin resistance was already reported not only in $P$. aeruginosa from bovine but also in Acinetobacter spp. from humans, which is worth noting because 
colistin has been considered as a limited antibiotic option for the treatment of $P$. aeruginosa and Acinetobacter spp. infections and is used as a 'last resort' in human medicine (Ko et al., 2007; Park et al., 2009). Given the importance of colistin and other antibiotics used as a limited antibiotic option, more careful usage of these antibiotics is essential.

After biofilm formation, biofilm status $P$. aeruginosa resisted antibiotics more strongly and resulted in notable changes in antibiotic susceptibility patterns. The MBEC of $P$. aeruginosa isolates differed from the MIC against all kinds of antibiotics tested, and the antibiotics applied at MIC failed to kill biofilm status $P$. aeruginosa. Even gentamicin, amikacin, meropenem and ciprofloxacin, which were effective against most of the planktonic status isolates in this study, failed to eradicate biofilm status isolates at the MIC. In the case of meropenem and ciprofloxacin, this tendency was remarkably evident from the particularly high MBEC/MIC ratio of the antibiotics. MBEC/MIC values for chloramphenicol, tetracycline and cefoxitin were relatively lower than those for other antibiotics. However, this result might be caused by the high MIC values rather than the low MBEC values, owing to the intrinsic resistance of $P$. aeruginosa to chloramphenicol, tetracycline and cefoxitin. These data suggested that antibiotics that are effective against planktonic status $P$. aeruginosa could fail to eradicate biofilm status $P$. aeruginosa isolates, and biofilm formation could be a critical factor resulting in the failure of antibiotic therapy due to the intrinsic resistance mechanisms of bacteria. The findings also suggest that MBEC as well as MIC should be reflected in the treatment process and that the MBEC/MIC ratio could help exclude antibiotics ineffective against biofilm status $P$. aeruginosa. In addition, the MBEC of other biofilm-forming bacteria associated with mastitis, such as Staphylococcus aureus, may help to increase treatment efficiency.

Our results indicate that most of the $P$. aeruginosa isolates originating from the raw milk of mastitis-affected dairy cows in Korea had a genotype that may be related to an increase in the SCCs. The efficiency of antibiotic therapy against bovine mastitis involving $P$. aeruginosa could be improved if data pertaining to MBEC values as well as MIC test results were applied together during treatment.

\section{Acknowledgements}

This research was supported by the Bio-industry Technology Development Program, Ministry for Food, Agriculture, Forestry and Fisheries, Republic of Korea (IPET 110032-3 and 311001-03-1-HD120) and by the National Research Foundation of Korea grants funded by the South Korean government (NRF-2010-1-E00057). 


\section{References}

CLSI (2012): Performance Standards for Antimicrobial Susceptibility Testing. 22nd Informational Supplement. M100-S22. Clinical and Laboratory Standards Institute, Wayne, PA, USA.

De Haas, Y., Barkema, H. W., Schukken, Y. H. and Veerkamp, R. F. (2005): Association between somatic cell count patterns and the incidence of clinical mastitis. Prev. Vet. Med. 67, 55-68.

Engel, J. and Balachandran, P. (2009): Role of Pseudomonas aeruginosa type III effectors in disease. Curr. Opin. Microbiol. 12, 61-66.

Fleiszig, S., Wiener-Kronish, J. P., Miyazaki, H., Vallas, V., Mostov, K. E., Kanada, D., Sawa, T., Yen, T. and Frank, D. W. (1997): Pseudomonas aeruginosa-mediated cytotoxicity and invasion correlate with distinct genotypes at the loci encoding exoenzyme S. Infect. Immun. 65, 579-586.

Hillerton, J. E. and Kliem, K. E. (2002): Effective treatment of Streptococcus uberis clinical mastitis to minimize the use of antibiotics. J. Dairy Sci. 85, 1009-1014.

Jung, S. C., Lim, S. K., Lee, H. S., Jung, B. Y., Lee, J. Y., Yang, C. B. and Shin, H. C. (2008): Antibiotics use in animal and resistant bacteria. Infect. Chemother. 40, 144-149.

Ko, K. S., Suh, J. Y., Kwon, K. T., Jung, S.-I., Park, K.-H., Kang, C. I., Chung, D. R., Peck, K. R. and Song, J.-H. (2007): High rates of resistance to colistin and polymyxin B in subgroups of Acinetobacter baumannii isolates from Korea. J. Antimicrob. Chemoth. 60, 1163-1167.

Ledbetter, E. C., Mun, J. J., Kowbel, D. and Fleiszig, S. M. J. (2009): Pathogenic phenotype and genotype of Pseudomonas aeruginosa isolates from spontaneous canine ocular infections. Invest. Ophthalmol. Vis. Sci. 50, 729-736.

Lyczak, J. B., Cannon, C. L. and Pier, G. B. (2000): Establishment of Pseudomonas aeruginosa infection: lessons from a versatile opportunist. Microbes Infect. 2, 1051-1060.

Magiorakos, A. P., Srinivasan, A., Carey, R. B., Carmeli, Y., Falagas, M. E., Giske, C. G., Harbarth, S., Hindler, J. F., Kahlmeter, G. and Olsson-Liljequist, B. (2012): Multidrugresistant, extensively drug-resistant and pandrug-resistant bacteria: an international expert proposal for interim standard definitions for acquired resistance. Clin. Microbiol. Infect. 18, 268-281.

Mah, T. F. C. and O'Toole, G. A. (2001): Mechanisms of biofilm resistance to antimicrobial agents. Trends Microbiol. 9, 34-39.

Melchior, M., Vaarkamp, H. and Fink-Gremmels, J. (2006): Biofilms: A role in recurrent mastitis infections? Vet. J. 171, 398-407.

Moon, J., Lee, A., Kang, H., Lee, E., Joo, Y., Park, Y. H., Kim, M. and Koo, H. (2007): Antibiogram and coagulase diversity in staphylococcal enterotoxin-producing Staphylococcus aureus from bovine mastitis. J. Dairy Sci. 90, 1716-1724.

Oliveira, M., Bexiga, R., Nunes, S., Carneiro, C., Cavaco, L., Bernardo, F. and Vilela, C. (2006): Biofilm-forming ability profiling of Staphylococcus aureus and Staphylococcus epidermidis mastitis isolates. Vet. Microbiol. 118, 133-140.

Park, Y., Koo, H., Kim, S., Hwang, S., Jung, W., Kim, J., Shin, S., Kim, R. and Park, Y. H. (2007): The analysis of milk components and pathogenic bacteria isolated from bovine raw milk in Korea. J. Dairy Sci. 90, 5405-5414.

Park, Y. K., Jung, S.-I., Park, K.-H., Cheong, H. S., Peck, K. R., Song, J.-H. and Ko, K. S. (2009): Independent emergence of colistin-resistant Acinetobacter spp. isolates from Korea. Diagn. Micr. Infec. Dis. 64, 43-51.

Roy-Burman, A., Savel, R. H., Racine, S., Swanson, B. L., Revadigar, N. S., Fujimoto, J., Sawa, T., Frank, D. W. and Wiener-Kronish, J. P. (2001): Type III protein secretion is associated with death in lower respiratory and systemic Pseudomonas aeruginosa infections. J. Infect. Dis. 183, 1767-1774.

Selezska, K., Kazmierczak, M., Musken, M., Garbe, J., Schobert, M., Haussler, S., Wiehlmann, L., Rohde, C. and Sikorski, J. (2012): Pseudomonas aeruginosa population structure revisited 
under environmental focus: impact of water quality and phage pressure. Environ. Microbiol. 14, 1952-1967.

Spilker, T., Coenye, T., Vandamme, P. and LiPuma, J. J. (2004): PCR-based assay for differentiation of Pseudomonas aeruginosa from other Pseudomonas species recovered from cystic fibrosis patients. J. Clin. Microbiol. 42, 2074-2079.

Sumathi, B. R., Veeregowda, B. M. and Gomes, A. R. (2008): Prevalence and antibiogram profile of bacterial isolates from clinical bovine mastitis. Vet. World 1, 237-238.

Szmolka, A., Cramer, N. and Nagy, B. (2012): Comparative genomic analysis of bovine, environmental and human strains of Pseudomonas aeruginosa. FEMS Microbiol. Lett. 335, 113-122.

Vance, R. E., Rietsch, A. and Mekalanos, J. J. (2005): Role of the type III secreted exoenzymes S, $\mathrm{T}$, and $\mathrm{Y}$ in systemic spread of Pseudomonas aeruginosa PAO1 in vivo. Infect. Immun. 73, $1706-1713$

Wiehlmann, L., Wagner, G., Gramer, N., Siebert, B., Gudowius, P., Morales, G., Kohler, T., Van Delden, C., Weinel, C. and Slickers, P. (2007): Population structure of Pseudomonas aeruginosa. Proc. Natl Acad. Sci. USA 104, 8101-8106. 